\title{
Thermophilic and non-thermophilic Campylobacter species Emits Distinct Volatile Organic Compounds in Different Culture Media and Growth Phases
}

Filipe Magnum Silva Dos Santos

Unviersiti Malaya

Kah Hin Low

Unviersiti Malaya

Lay Ching Chai ( $\sim$ lcchai@um.edu.my )

Unviersiti Malaya

Research Article

Keywords:

Posted Date: January 13th, 2022

DOI: https://doi.org/10.21203/rs.3.rs-1247479/v1

License: (c) (i) This work is licensed under a Creative Commons Attribution 4.0 International License.

Read Full License 


\section{Abstract}

Bacteria emits a multitude of volatile organic compounds (VOCs) into the headspace as a mean of interactions with the environments, as well as intra- and interkingdom communication for survival and persistence in the nature and within their hosts. Campylobacter, which is often found in poultry and ruminants, has shown great persistence in aquatic environments, making it one of the world's most dangerous foodborne pathogens, killing thousands of people annually. In this study, the VOCs emitted by both thermophilic (C. jejuni, $C$. coli and $C$. lari) and non-thermophilic Campylobacter (C. fetus) of clinical concerns, impacted by nutrients composition (media) and growth phase were identified. Most thermophilic Campylobacter were shown to release volatile alcohols and ketones $(1 \mathrm{~s}, 4 \mathrm{R}, 7 \mathrm{R}, 11 \mathrm{R}-1,3,4,7-$ Tetramethyltricyclo $[5.3 .1 .0(4,11)]$ undec-2-en-8-one and Isophorone) during early stationary and stationary phases using active sampling with active charcoal adsorbent and GC-MS analysis. C. jejuni cultured in the Brain Heart Infusion had 1-Heptadecanol in its headspace gas, but not in Bolton Broth. The non-thermophilic $C$. fetus did not produce alcohols or ketones, but rather a variety of unidentified chemicals that will require further investigation in the future. Overall, PCA analysis revealed that the five Campylobacter strains studied created distinct volatilomes, allowing for future Campylobacter identification based on VOCs.

\section{Introduction}

The genus Campylobacterhas been known for its challenging detection, isolation and identification in the laboratory, due to its physiological characteristics, such as non-glycolytic diet, low-oxygen requirement to grow (obligate microaerophile), and ability to enter in a coccoid form during non-favourable growth conditions, known as viable but non-culturable (VBNC) state ${ }^{1,2,3}$. However, despite the constraints of culturing this microorganism in vitro, Campylobacter spp. are ubiquitous in nature and colonise the intestinal tracts of many wild and domesticated animals ${ }^{4,5}$. These foodborne pathogens only require a small number of bacterial cells to cause human infection ${ }^{5}$, which is primarily transmitted through the consumption of poultry meat, particularly contaminated broiler chicken (Gallus gallus) ${ }^{2,6}$.

Campylobacter jejuni subsp. jejuni is the major species of the taxon and it is recognised as the leadingcause of human gastroenteritis worldwide ${ }^{7,8,9}$. The infection triggered by the pathogen is mainly characterised by mild and self-limiting symptoms, including watery to bloody diarrhoea, abdominal pain, fever, and vomiting ${ }^{2,10}$. However, severe sequalae may occur, such as Guillain-Barré syndrome, an autoimmune disease characterised by weakness of limbs and paralysis ${ }^{2,10,11}$. On the other hands, $C$. coli is the second most reported species of human campylobacteriosis. It is very similar to $C$. jejuni, and frequently isolated together with the $C$. jejuni from the contaminated poultry and faeces samples ${ }^{12,13}$. The third most clinically-significant Campylobacter species is $C$. lari. Although $C$. lari is infrequently associated with human infections, it has been isolated from patients with diarrhoea ${ }^{14,12}$. Another clinically important species is Campylobacter fetus subsp fetus that is an opportunistic human pathogen, that primarily affects patients with weakened immune system ${ }^{15,12}$. 
The standard food safety testing is mainly based on culturing, polymerase chain reaction (PCR), and enzyme-linked immunosorbent assays (ELISA) approach that is time-consuming, labour-intensive, and prone to false negative results $3,16,17,18$. To meet the demand for an increasing number of food testing for global food trade, new method for Campylobacter detection in foods that is simpler, rapid, and highly sensitive is required urgently ${ }^{10}$. In this regards, a number of studies have demonstrated that bacteria produce distinctive volatiles organic compounds (VOCs) during culturing, create a single VOC-emission pattern that could potentially be developed into a unique metabolic biomarker for bacterial identification with high specificity, simplicity and in real-time $19,20,21,22$.

The bacterial VOCs are detected by means of gas chromatographic techniques, particularly the gas chromatography-mass spectrometry (GC-MS), which is generally preceded by a sample preparation ${ }^{19,23}$. For instance, solid phase microextraction (SPME) has been largely used for sampling microbial VOCs $^{19,24}$. However, owing to the lack of standardized methods, the nature of targeted sample matrix (i.e., the composition and the analytes' properties), the experimental requirements and constraints (i.e., costs and instrumental issues, etc.), select the most suitable sampling procedure to serve the purpose of Voc fingerprinting is challenging $25,23,26$. Nevertheless, sorption tube approach seems to be a suitable strategy that is widely adopted for gas sampling as it could retain a range of compatible VOCs at ambient temperature subjected to the interaction between the analytes and the sorbent material; the retained content could be desorbed for subsequent measurement ${ }^{25,26}$. This technique is simple, inexpensive, and more appropriate for thermally unstable compounds, when desorption via compatible solvent system is applied $^{27,28,24}$.

Moreover, in contrast with the research attention that bacterial VOCs have drawn lately ${ }^{19,29,30}$, only few studies regarding to VOC-emissions of Campylobacter species have been published. The whole-cell fatty acids of Campylobacter fetus subsp. venerealis was investigated in a study that found hexadecanoic acid as the most significant volatile emitted by the bacterium ${ }^{31}$. Also, the profile of VoCs emitted by $C$. jejuni in contaminated human stools and by $C$. jejuni and $C$. coli in chicken faeces was previously explored $^{32,33}$. Additionally, the prevalence of alcohols were observed in broth inoculated with $C$. jejuni after 20 hours of incubation ${ }^{34}$. However, when subjected to the type of media, the growth phase, bacterial strain, and sampling setting, the VOCs emitted by a particular bacterial species could vary considerably $25,19,26,35$. Hence, this study attempted to investigate the variation of VOCs emitted by the four clinically significant species of Campylobacter (C. jejuni, $C$. coli, $C$. lari and $C$. fetus) in different culture media and growth phase. This research would be a cornerstone for further development of VOCbased sensing technology for detection of foodborne Campylobacter spp. in food.

\section{Results}

Identification of putative bacterial volatiles produced by Campylobacter 
Many peaks were detected during the GC-MS analysis of Campylobacter species. Figure 1 shows the similarity of the chromatograms of the bacterial samples and the blank media (negative control), demonstrating the complexity in identifying the putative biomarkers. Overall, the five Campylobacter strains included in this study were found to produce a total of 38 to 68 identified VOCs during growth in either Bolton or Brain Heart Infusion broth; of these VOCs identified, only 15.7 to $41.3 \%$ remained after removing the VOCs found present in the blank samples (i.e. VOCs produced by the culture media) and 3.1 to $10.8 \%$ of these VOCs were detected in at least two biological replicates (filtering criteria 2) (Table 1). The VOCs that passed both the filtering criteria 1 and 2 were then labelled as the putative VOC biomarkers.

Table 1

Number of VOCs detected in the bacterial samples by GC-MS and application of the filtering criteria.

\begin{tabular}{|c|c|c|c|c|c|c|}
\hline Strain & $\begin{array}{l}\text { Culture } \\
\text { Media }\end{array}$ & $\begin{array}{l}\text { Detected } \\
\text { GC } \\
\text { Peaks }\end{array}$ & $\begin{array}{l}\text { Identified } \\
\text { VOCs } \\
\text { (MS+RI) }^{\mathrm{a}}\end{array}$ & $\begin{array}{l}\text { VOCs } \\
\text { Pass } \\
\text { Criteria } \\
1^{b}\end{array}$ & $\begin{array}{l}\text { VOCs } \\
\text { Pass } \\
\text { Criteria } \\
2^{c}\end{array}$ & $\begin{array}{l}\text { Putative } \\
\text { discriminan } \\
\text { VOCs }\end{array}$ \\
\hline \multirow{2}{*}{$\begin{array}{l}\text { C. jejuni subsp jejuni } \\
\text { ATCC29428 }\end{array}$} & $\begin{array}{l}\text { Bolton } \\
\text { broth }\end{array}$ & 566 & 46 & $\begin{array}{l}9 \\
(19.5 \%)\end{array}$ & $\begin{array}{l}5 \\
(10.8 \%)\end{array}$ & 5 \\
\hline & $\begin{array}{l}\text { BHI } \\
\text { broth }\end{array}$ & 531 & 40 & $\begin{array}{l}14 \\
(35 \%)\end{array}$ & $2(5 \%)$ & 2 \\
\hline \multirow{2}{*}{$\begin{array}{l}\text { C. jejuni subsp jejuni } \\
\text { ATCC33291 }\end{array}$} & $\begin{array}{l}\text { Bolton } \\
\text { broth }\end{array}$ & 575 & 63 & $\begin{array}{l}13 \\
(20.6 \%)\end{array}$ & $\begin{array}{l}2 \\
(3.1 \%)\end{array}$ & 2 \\
\hline & $\begin{array}{l}\text { BHI } \\
\text { broth }\end{array}$ & 553 & 46 & $\begin{array}{l}19 \\
(41.3 \%)\end{array}$ & $\begin{array}{l}5 \\
(10.8 \%)\end{array}$ & 5 \\
\hline $\begin{array}{l}\text { Campylobacter coli } \\
\text { ATCC43478 }\end{array}$ & $\begin{array}{l}\text { Bolton } \\
\text { broth }\end{array}$ & 545 & 38 & $\begin{array}{l}6 \\
(15.7 \%)\end{array}$ & $\begin{array}{l}2 \\
(5.2 \%)\end{array}$ & 2 \\
\hline $\begin{array}{l}\text { Campylobacter fetus subs. } \\
\text { Fetus ATCC } 24374\end{array}$ & $\begin{array}{l}\text { Bolton } \\
\text { broth }\end{array}$ & 601 & 43 & $\begin{array}{l}9 \\
(20.9 \%)\end{array}$ & $\begin{array}{l}3 \\
(7.0 \%)\end{array}$ & 3 \\
\hline $\begin{array}{l}\text { Campylobacter lari } \\
\text { ATCC35221 }\end{array}$ & $\begin{array}{l}\text { Bolton } \\
\text { broth }\end{array}$ & 432 & 68 & $\begin{array}{l}16 \\
(23.5 \%)\end{array}$ & $\begin{array}{l}4 \\
(5.8 \%)\end{array}$ & 4 \\
\hline \multicolumn{7}{|c|}{$\begin{array}{l}\text { a Identified VOC is compound that its identity has been confirmed by its Mass Spectrum (MS) and } \\
\text { Retention Index (RI). }\end{array}$} \\
\hline \multicolumn{7}{|c|}{${ }^{b}$ Criteria 1: VOC that is not found in the background (blank media). } \\
\hline
\end{tabular}

A total of 16 VOC biomarkers were identified to be associated with Campylobacter species (Figure 2; Supplementary data A1). These VOCs were found to belong to alcohol (3), ketone (2), ester (1), phenyl alkene (1), phenol (1) and unidentified (8) (Figure 1). Some of the putative biomarkers were labelled as "not identified" due to a low matching with the mass spectrum and retention index. However, these 
unidentified compounds were labelled according to their mass spectrum and calculated retention index for comparison between samples.

It is interesting to point out that there was no overlapping in the VOC biomarkers emitted by Campylobacter species cultured in Bolton and BHI broth (Figure 2). Both strains of Campylobacter jejuni (ATCC 29428 and ATCC 33291) emitted 1s,4R,7R,11R-1,3,4,7-Tetramethyltricyclo [5.3.1.0(4,11)] undec-2en-8-one (ketone) when cultured in Bolton broth; while emitted 1-Heptadecanol (alcohol) when they were cultured in BHI broth (Figure 2).

While both $C$. jejuni strains shared only 1 similar VOC biomarkers growing in Bolton broth, $C$. jejuni ATCC 29428 shared more VOC biomarkers with C. lari ATCC 35221: 3-Dodecanol, 3,7,11-trimethyl- (alcohol), 1s,4R,7R,11R-1,3,4,7-Tetramethyltricyclo [5.3.1.0(4,11)] undec-2-en-8-one (ketone) and not identified 29 (Figure 2). The VOC not identified 29 was in fact found to be emitted also by C. fetus ATCC 27374. Another ketone, isophorone was emitted by $C$. jejuni ATCC 33291 and C. coli 43478. It is worthy to note that $C$. coli did not share similarity with $C$. fetus and $C$. lari, in term of VOC biomarkers (Figure 2).

\section{Bacterial Growth}

The growth curve of Campylobacter species cultured in both Bolton and $\mathrm{BHI}$ broth were determined by MPN enumeration approach at $0 \mathrm{~h}, 12 \mathrm{~h}, 24 \mathrm{~h}$, and $48 \mathrm{~h}$ of incubation. The growth curve was then plotted (Figure 3). All, but $C$. jejuni 33291 in BHI broth showed exponential growth in the first 12 hours of incubation, indicating of the exponential growth phase; which was then followed by a significant deceleration in the growth rate at $12 \mathrm{~h}$ to $24 \mathrm{~h}$ and $48 \mathrm{~h}$, indicating the late exponential phase entering into early phase of stationary growth phase (Figure 3). For C. jejuni 33291 cultivated in BHI broth, the exponential phase continued for another 12 hours before the growth rate started to slow down at 24 hours (Figure 2d).

\section{Volatile organic compounds emitted by Campylobacter species during different growth phases}

Majority of the identified putative VOC-markers were detected after 12 hours of incubation, suggesting that the active production VOCs occur during the late exponential and early stationary phase of Campylobacterspp. under laboratory culturing conditions (Table 2). Generally, alcohol was detected in Campylobacter jejuni cultures at 24 hours and beyond; 1-Heptadecanol, which was only detected in $C$. jejuni ATCC 29428 BHI cultures was also detected after 12 hours of incubation (Table 2). Volatile ketones, 1s,4R,7R,11R-1,3,4,7-Tetramethyltricyclo [5.3.1.0(4,11)] undec-2-en-8-one and Isophorone, were mainly detected in the stationary phase at 48 hours (Table 2). 
Table 2

Relative peak area (average) of bacterial VOCs detected at 12,24 and 48 hours of incubation at $42^{\circ} \mathrm{C}$.

\begin{tabular}{|c|c|c|c|c|c|c|}
\hline \multirow[t]{3}{*}{ Strain } & \multirow[t]{3}{*}{ Media } & \multirow[t]{3}{*}{ Class } & \multirow[t]{3}{*}{ VOC } & \multicolumn{3}{|c|}{ Relative peak area } \\
\hline & & & & \multirow{2}{*}{$\begin{array}{l}\text { Exponential } \\
12 \mathrm{~h}\end{array}$} & \multicolumn{2}{|c|}{ Stationary } \\
\hline & & & & & $24 \mathrm{~h}$ & $48 \mathrm{~h}$ \\
\hline \multirow{8}{*}{$\begin{array}{l}\begin{array}{l}\text { C. jejuni subsp } \\
\text { jejuni }\end{array} \\
\text { ATCC } 29428\end{array}$} & \multirow{5}{*}{$\begin{array}{l}\text { Bolton } \\
\text { broth }\end{array}$} & Alcohol & 1,8-Nonadien-3-ol & 0 & 0.1075 & 0 \\
\hline & & Alcohol & $\begin{array}{l}\text { 3-Dodecanol, 3,7,11- } \\
\text { trimethyl- }\end{array}$ & 0 & 0.102 & $0.0458^{*}$ \\
\hline & & Ketone & $\begin{array}{l}\text { 1s,4R,7R,11R-1,3,4,7- } \\
\text { Tetramethyltricyclo } \\
\text { [5.3.1.0(4,11)] undec- } \\
\text { 2-en-8-one }\end{array}$ & 0.0478 & 0 & 0.0592 \\
\hline & & Unknown & Not Identified - 29 & 0 & 0 & 0.122 \\
\hline & & Unknown & Not Identified - 16 & 1.324 & 0 & 0 \\
\hline & \multirow{3}{*}{$\begin{array}{l}\mathrm{BHI} \\
\text { broth }\end{array}$} & Alcohol & 1-Heptadecanol & 0 & 0.0416 & 0.3796 \\
\hline & & Ketone & Isophorone & 0 & 0 & $0.2128^{*}$ \\
\hline & & Unknown & Not Identified - 85 & 0 & 0 & 0.0589 \\
\hline \multirow{2}{*}{$\begin{array}{l}\text { C. jejuni subsp } \\
\text { jejuni } \\
\text { ATCC } 33291\end{array}$} & \multirow{2}{*}{$\begin{array}{l}\text { Bolton } \\
\text { broth }\end{array}$} & Ketone & Isophorone & 0 & 0 & 0.2319 \\
\hline & & Ketone & $\begin{array}{l}\text { 1s,4R,7R,11R-1,3,4,7- } \\
\text { Tetramethyltricyclo } \\
\text { [5.3.1.0(4,11)] undec- } \\
\text { 2-en-8-one }\end{array}$ & 0 & 0.0345 & 0 \\
\hline $\begin{array}{l}\text { C. jejuni subsp } \\
\text { jejuni }\end{array}$ & \multirow[t]{6}{*}{$\begin{array}{l}\text { BHI } \\
\text { broth }\end{array}$} & Alcohol & 1-Heptadecanol & 0 & 0.0226 & $0.0314^{*}$ \\
\hline \multirow[t]{5}{*}{ ATCC 33291} & & Ketone & Isophorone & 0 & 0 & $0.2991^{*}$ \\
\hline & & Ester & Diisobutyl phthalate & $0.5166^{*}$ & 0.0647 & 0.0671 \\
\hline & & $\begin{array}{l}\text { Phenyl } \\
\text { Alkene }\end{array}$ & $\begin{array}{l}\text { Benzene, (1- } \\
\text { propyloctyl)- }\end{array}$ & 0 & 0.03625 & 0 \\
\hline & & Unknown & Not Identified - 73 & 0.1580 & 0 & 0 \\
\hline & & Unknown & Not Identified - 84 & 0.1994 & 0 & 0 \\
\hline \multirow{2}{*}{$\begin{array}{l}\text { Campylobacter } \\
\text { coli }\end{array}$} & \multirow{2}{*}{$\begin{array}{l}\text { Bolton } \\
\text { broth }\end{array}$} & Ketone & Isophorone & 0 & 0 & 0.2770 \\
\hline & & Phenol & $\begin{array}{l}\text { Phenol, 3,5-bis(1,1- } \\
\text { dimethylethyl)- }\end{array}$ & 0 & 0 & 5.80 \\
\hline
\end{tabular}

* VOC detected in only one bacterial sample. 


\begin{tabular}{|c|c|c|c|c|c|c|}
\hline \multirow{4}{*}{$\begin{array}{l}\text { Campylobacter } \\
\text { fetus subs. } \\
\text { Fetus }\end{array}$} & \multirow{4}{*}{$\begin{array}{l}\text { Bolton } \\
\text { broth }\end{array}$} & Unknown & Not Identified - 29 & 25.79 & 15.28 & 0 \\
\hline & & Unknown & Not Identified - 40 & 0 & 16.55 & 0 \\
\hline & & Unknown & Not Identified - 58 & 0 & 2.26 & 0 \\
\hline & & Unknown & Not Identified - 42 & 0 & 5.07 & 0 \\
\hline \multirow[t]{4}{*}{$\begin{array}{l}\text { Campylobacter } \\
\text { lari }\end{array}$} & \multirow[t]{4}{*}{$\begin{array}{l}\text { Bolton } \\
\text { broth }\end{array}$} & Alcohol & $\begin{array}{l}\text { 3-Dodecanol, 3,7,11- } \\
\text { trimethyl- }\end{array}$ & 0 & 0 & 6.25 \\
\hline & & Ketone & $\begin{array}{l}\text { 1s,4R,7R,11R-1,3,4,7- } \\
\text { Tetramethyltricyclo } \\
\text { [5.3.1.0(4,11)] undec- } \\
\text { 2-en-8-one }\end{array}$ & 0 & 0 & 6.64 \\
\hline & & Unknown & Not Identified - 25 & 0 & 10.40 & 0 \\
\hline & & Unknown & Not Identified - 29 & 0 & 0 & 13.3 \\
\hline
\end{tabular}

\section{Discussion}

The measurement results of bacterial VOCs depend greatly on the analytical methods adopted for fingerprinting, especially the gas sampling strategy. Although the charcoal-based matrix is a sufficiently suitable adsorbent for a wide range of compounds ${ }^{27}$, one of the major drawbacks of using the charcoal adsorbent used in this study was the loss of volatiles, which could be caused by the solvent dilution factor, lesser affinity with polar compounds, and overload of the sorbent matrix ${ }^{27,28,24}$. These might explain the detection of less putative VOC-markers in this study as compared to other reported study that used different adsorbents ${ }^{34}$ and technique such as thermal desorption ${ }^{28,35}$. However, the charcoal adsorbent has been proven to perform sufficiently well to detect the most predominant bacterial volatiles that were commonly reported by other researchers, such as alcohols and ketones.

In this study, alcohols were the most commonly detected VOCs in Campylobacter spp., produced mainly in the early stationary and stationary growth phase (Table 2). The detected alcohol-based VOCs included the fatty alcohols 3,7,11-trimethyl-3-dodecanol and 1-heptadecanol, as well as the non-cyclic alkene alcohol 1,8-nonadien-3-ol (Figure 2). The probable pathways for bacterial synthesis of fatty alcohol and long-chain alcohols are through hydrogenation of methyl esters of fatty acids ${ }^{36}$, as well as by the $\beta$ - or aoxidation of fatty acid by-products ${ }^{37}$. For instance, the production of fatty alcohols in $E$. coli begins with a thioesterase-mediated conversion of a fatty acyl-ACP (acyl carrier protein) to a free fatty acid. The free fatty acid is then converted to a fatty acyl-CoA by a fatty acyl-CoA synthase. The resulting fatty acyl-CoA can subsequently be metabolised via the $\beta$ - oxidation route or reduced to its corresponding fatty alcohol by the generation of its corresponding fatty aldehyde in a NADPH-dependent fatty acyl-CoA reductasecatalysed process ${ }^{38}$. Additionally, 1,8-nonadien-3-ol has been reported to be emitted by Pseudomonas putida as an antimicrobial volatile against plant pathogens ${ }^{39}$, and commercial fermentation starters 
during food fermentation ${ }^{40}$. On the other hand, 1-heptadecanol was also found to be one of the antifungal volatiles emitted by Pseudomonas spp. isolated from canola and soybean ${ }^{41}$.

The two most predominantly isolated volatile ketones emitted by Campylobacter spp. in the current study were isophorone and 1s,4R,7R,11R-1,3,4,7-tetramethyltricyclo [5.3.1.0(4,11)] undec-2-en-8-one (Figure 2 and Table 2). The ketones were found to be emitted by the thermophilic Campylobacter (C. jejuni, $C$. coli and $C$. lari) in both Bolton broth and Brain Heart Infusion culture (Table 2). However, it is noteworthy to point out that ketones were detected generally after 12 hours of incubation at 24 and 48 hours, corresponding to the early phase of stationary and stationary growth phase of Campylobacter spp. in vitro (Table 2). Similarly, Reese et al. ${ }^{35}$ also reported on the observation of detection of ketones with long carbon chains in the stationary phase and beyond. Isophorone is an unsaturated cyclic ketone that has a peppermint-like smell, and it evaporates faster than water ${ }^{42}$. It can be naturally found in cranberries ${ }^{42}$, honey ${ }^{43}$ and as a beetle pheromone ${ }^{44}$. Also, it has been already reported by Schulz and Dickschat ${ }^{45}$ as a volatile emitted by the cyanobacteria Oscillatoria perornata. Whereas $1 \mathrm{~s}, 4 \mathrm{R}, 7 \mathrm{R}, 11 \mathrm{R}-1,3,4,7-$

tetramethyltricyclo $[5.3 .1 .0(4,11)]$ undec-2-en-8-one is sesquiterpene ketones occurs naturally in fungi and plants ${ }^{46}$ and it has an important antibiotic role as a bacterial secondary metabolite ${ }^{47} .1 \mathrm{~s}, 4 \mathrm{R}, 7 \mathrm{R}, 11 \mathrm{R}-$ 1,3,4,7-tetramethyltricyclo [5.3.1.0 $(4,11)]$ undec-2-en-8-one has been also reported as one the volatiles of lignin isolated from oil palm empty fruit ${ }^{48}$.

Generally, the biosynthesis pathway of ketones, particularly methyl ketones, involve the decarboxylation of fatty acids ${ }^{49}$. However, isophorone and $1 \mathrm{~s}, 4 \mathrm{R}, 7 \mathrm{R}, 11 \mathrm{R}-1,3,4,7$-tetramethyltricyclo $[5.3 .1 .0(4,11)]$ undec-2en-8-one are terpenoids with a molecule of oxygen as the functional group of a ketone. Hence, the bacterial metabolic routes of terpenoids, unlike methyl ketones, are synthesised from the five-carbon building blocks, isopentenyl diphosphate (IDP) and dimethylallyl diphosphate (DMADP). These molecules are likely to be originated from pyruvate and glyceraldehyde-3-phosphate in the methylerythritol-4phosphate (MEP) and the Mevalonate (MVA) pathways ${ }^{50}$.

The presence of alcohols and ketones in the VOC profile of $C$. jejuni is in accordance with other studies that observed the prevalence of these compounds in the cultures of $C$. jejuni and in matrices contaminated by the bacterium. Probert et al. ${ }^{32}$ also noticed the presence of these chemicals in stool of patients contaminated with $C$. jejuni. Alcohols and ketones were detected as discriminant VOCs of chicken faeces contaminated with $C$. jejuni and $C$. coli ${ }^{33}$. Alcohols and ketones were also abundant in the bacterial samples of $C$. jejuni (ATCC 33560) cultivated in BHI broth after 20 hours of incubation ${ }^{34}$.

The VOC profiling of other clinically important Campylobacter species, such as C. coli, C. fetus subsp. fetus and $C$. lari, was a pioneering step towards the characterization of the volatilome of these pathogens. Isophorone and 3,5-bis(1,1-dimethylethyl)-phenol were discriminant volatiles emitted by $C$. coli., 3,7,11-trimethyl-3-dodecanol and 1s,4R,7R,11R-1,3,4,7-tetramethyltricyclo [5.3.1.0(4,11)] undec-2-en8-one were identified as biomarkers of $C$. lari. However, the profile of $C$. fetus consisted of only notidentified compounds, that despite of their unconfirmed identity, these compounds were important to 
demonstrate the distinction of $C$. fetus among the other species. Nonetheless, future research should be able to widen the identification of the volatiles of these foodborne bacteria by employing more sensitive VOC-analytical methods.

The PCA analysis demonstrated clear distinguishable markers based on the VOCs profile emitted by the various strains of Campylobacter spp. included in this work; as well as VOCs profiles that is growth phase dependent (Figure 4 and Figure 5). PCA has been used by numerous researchers to identify similarity and variation in the multivariable dataset, such as Milanowski and co-workers ${ }^{51}$ that has demonstrated discrimination of salivary bacteria (Hafnia alvei, Pseudomonas luteola and Staphylococcus warneri) based on their volatiles' profiles; and discrimination of $F$. tularensis subsp. novicida, Bacillus anthracis Sterne and Bacillus anthracis Ames in the study by Reese and co-workers ${ }^{35}$.

The study of the variation in the VOCs emitted by the various clinically important Campylobacter spp. including Campylobacter jejuni subsp. jejuni in vitro via active sampling with an activated charcoal sorbent, revealed the culture- and growth phase-associated volatilome profiles of thermophilic and nonthermophilic Campylobacter spp. The findings will provide an insight into the potential roles of bacterial VOCs in their growth and survival in various niches in environment and its host. The findings may also provide a baseline to development of VOC-based detection method of the fastidious Campylobacter spp. Nevertheless, more in-depth studies are required to identify the unknown VOCs detected in this study. Also, it will be meaningful to investigate other influencing factors, such as coculturing of Campylobacter $s p p$. with other bacteria population to fully understand the mechanisms and role of bacterial VOCs.

\section{Methods}

\section{Strains, Culture Media, and Laboratorial Maintenance}

In this study, five bacterial strains of Campylobacter genus were targeted: Campylobacter jejuni subsp. jejuni (ATCC 33291 and ATCC 29428), Campylobacter coli (ATCC 43478), Campylobacter fetus subsp. fetus (ATCC 27374), and Campylobacter lari (ATCC 35221). These strains were acquired from Microbiologics (Minnesota, USA). The strains of Campylobacter spp. were revived by inoculating the swab into sterile Bolton broth (BB; Oxoid, UK) supplemented with $5 \%$ of lysed horse blood. The lysed horse blood was prepared from fresh horse blood, which was purchased from the Faculty of Veterinary Medicine, Universiti Putra Malaysia. The fresh horse blood was subjected to multiple rounds of freezing and thawing until no separation into two layers was observed ${ }^{53}$. The inoculated media were incubated at $37 \mathbb{Q C}$ for $48 \mathrm{~h}$ in a sealed universal bottle with $20 \%$ of headspace, creating the microaerophilic condition.

After 48 hours of incubation, the turbid broth was aliquoted into sterilized Eppendorf tubes $(1.5 \mathrm{ml}$ of volume) and spun down by centrifugation at 6,500 RPM (Rotation Per Minute) for 2 minutes. The supernatant was discarded and $0.5 \mathrm{ml}$ of double strength Brain Heart Infusion broth (BHI; Himedia, India) and $0.5 \mathrm{ml}$ glycerol were added into the vials, then it was thoroughly mixed by pipetting. The glycerol stocks were then divided into two parts to be maintained in the freezers at $-20 \otimes C$ and $-80 \otimes C$. 
The strains of Campylobacter were revived from glycerol stocks for the headspace-air sampling as follows: one hundred microliters were transferred from the glycerol vial to a sterile Bolton broth tube

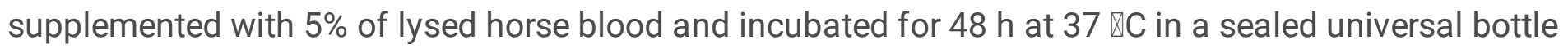
with $20 \%$ of headspace. After the growth, the inoculum was streaked onto modified Charcoal Cefoperazone Deoxycholate Agar (mCCDA; Oxoid, UK) in triplicate and incubated under microaerophilic atmosphere generated by Campygen $3.5 \mathrm{~L}$ (Oxoid, Basingstoke Hampshire, UK) at $37 \mathbb{} \mathrm{C}$ for $48 \mathrm{~h}$. Five to ten colonies were then transferred to a test tube containing BB and gently mixed. After that, $1 \mathrm{ml}$ of the bacterial suspension was pipetted into a $100 \mathrm{ml}$ screw cap bottle (Duran, Mainz, Germany) containing 80 $\mathrm{ml}$ of culture media and leaving $20 \mathrm{ml}$ of headspace volume for the headspace gas sampling and VOC detection.

\section{Headspace-air Sampling And Experimental Conditions}

Briefly the VOCs were collected by forcing the headspace gas in the bacteria culture bottle to pass through an ORBO activated coconut charcoal $(100 / 50 \mathrm{mg})$ adsorbent tube $(6 \times 70 \mathrm{~mm})$ (Merck, Darmstadt, Germany) at a fixed rate by a pump. The adsorbent tube was then removed, sealed, and pending for solvent extraction and subsequent GC-MS analysis. However, preliminary tests were performed to adjust the volume of headspace-air that needed to pass through the adsorbent matrix to ascertain detectable signals for subsequent investigations. The apparatus used for the sampling was assembled inside a anaerobic jar with a lit candle to generate the microaerophilic condition. The sample bottle was equipped with a GL 45 screw cap with two ports (Duran, Mainz, Germany). Both inlet and outlet ports were connected by Tygon tubes $(4.0 \mathrm{~mm}$ of internal diameter). The inlet tube was connected to a portable battery air pump DC-900 (Xilong, China) operated at a flow rate of approximately $2 \mathrm{~L} / \mathrm{min}$ and the sorbent tube was inserted in the outlet tube on the other hand. The duration of the headspace-air sampling was according to the length of each incubation period.

The C. jejuni strains (ATCC 29428 and 33291) were inoculated individually in two different high-protein media: BB supplemented with $5 \%$ of lysed horse blood and double strength $\mathrm{BH}$; with initial concentration of approximately $10^{4} \mathrm{MPN} / \mathrm{ml}$. All the samples were incubated at $37 \llbracket \mathrm{C}$ and the headspace air was sampled during three time-points: $12 \mathrm{~h}, 24 \mathrm{~h}$, and $48 \mathrm{~h}$. The others Campylobacter species: $C$. coli, $C$. fetus subs. fetus and $C$. lari were inoculated only in BB supplemented with $5 \%$ of lysed horse blood and with initial bacterial concentration of approximately $10^{5}, 10^{3}$, and $10^{3} \mathrm{MPN} / \mathrm{ml}$, respectively. They were also incubated at $37 \rrbracket \mathrm{C}$ with continuous active headspace-air sampling for $12 \mathrm{~h}, 24 \mathrm{~h}$, and $48 \mathrm{~h}$.

\section{Solvent Desorption And Gc-ms Analysis}

After the headspace air sampling, the sorbent tube was removed, and the sorbent content was transferred into a $1.5 \mathrm{ml}$ vial. Then, $1 \mathrm{ml}$ of carbon disulfide (Merck, Darmstadt, Germany) was added into the vial for 
desorption of the VOCs. The vial was thoroughly shaken using a vortex mixer, and the extract was further transferred into another clean GC vial before sending for immediate GC-MS analysis ${ }^{27,28}$.

The carbon disulfide extract was analysed under splitless mode using a GC-MS (Brand: Shimadzu; Model: QP2010 ULTRA 7890A GC/MS Agilent 5975) based on the following settings: a capillary column RTX-5 MS (length: $30 \mathrm{~m}$, internal diameter: $0.25 \mathrm{~mm}$, and film thickness: $0.25 \mu \mathrm{m}$ ) was used; the temperature of the injection port was at $230^{\circ} \mathrm{C}$; the oven was held at $40^{\circ} \mathrm{C}$ for $1 \mathrm{~min}$, then the temperature was raised to $280^{\circ} \mathrm{C}$ at a rate of $6^{\circ} \mathrm{C}$ min-1, and the final temperature was held for $1 \mathrm{~min}$; a solvent cut of 3 min was performed and the length of the analysis was 25 min per sample.

For the samples containing $\mathrm{BHI}$ broth, the temperature program was further refined as the preliminary results indicated the need to improve the data collection. In this case, the temperature of the injection port was increase to $250^{\circ} \mathrm{C}$; similarly, the oven was held at $40^{\circ} \mathrm{C}$ for $1 \mathrm{~min}$, then the temperature was raised to $250^{\circ} \mathrm{C}$ at a rate of $6^{\circ} \mathrm{C}$ min-1, and the final temperature was held for 11 minutes; leading to a 46 min run time. For both settings, a full scan mode was used for the mass spectrometry analysis.

For referencing purposes, a series of n-alkanes standard (Merck, Darmstadt, Germany) was run under the exact same GC-MS operating conditions; the retention index calculated from the alkane runs is rather system independent and thus reproducible ${ }^{54}$.

\section{Enumeration Of Bacterial Count}

Before the incubation periods $(12,24$, and 48 hours) and after the headspace air sampling $1 \mathrm{ml}$ of the enrichment broth was collected from the sample for three-tube Most Probable Number (MPN) analysis. The aliquot was serially diluted using fresh BB with $5 \%$ of lysed horse blood in a 10 -fold dilution until maximum $10^{-9}$, or depending on the expected growth phase, and incubated in triplicate at $37 \mathbb{\mathrm { C }}$ for $48 \mathrm{~h}$ under microaerophilic condition ${ }^{53}$.

The tubes were then checked for turbidity. The tubes that became turbid were plated on mCCDA and incubated for $48 \mathrm{~h}$ at $37 \mathbb{} \mathrm{C}$ under microaerophilic condition to confirm the presence of Campylobacter in the tubes. The concentration of bacteria cells in the tube (MPN/ml) was then calculated with a MPN calculator developed by the US governmental environmental protection agency (EPA) ${ }^{55}$. Also, the growth curve of Campylobacter species (MPN/ml) for all replicates throughout the experiment was then plotted.

\section{Data Analysis}

The VOCs were identified by taking both mass spectra and retention index into account. The mass spectrum of each compound was matched against the reference spectrum available in NIST mass spectral library, only those with high degree of similarity $(>70 \%)$ were further considered ${ }^{56}$. In addition to library mapping, GC retention index (RI) provide complementary information to aid the identification of VOCs. The normalized retention time (non-isothermal Kovats retention index) was calculated according to 
the formula of Van Den Dool \& Kratz ${ }^{57}$. For further identification, the calculated RIs were compared with the reference Rls of same kind of stationary phase; those with $5 \%$ or more of relative standard deviation were rejected ${ }^{35}$.

Moreover, two criteria were adopted here to decide the putative volatiles, that are most likely produced by the microorganism, with relative consistency (see Results, Table 1). First criterion, the compound is not present in the background (blank media) at the same timepoint (incubation period) of the bacterial samples, and second criterion, the compound was detected in at least two samples throughout the repetitions. Only filtered VOCs were considered for statistical comparison of bacterial strains and growth phases. The relative peak area of these selected compounds was subjected to PCA to explore the similarities/dissimilarities in VOCs patterns that might associated with the bacterial strains, the production of VOC during different growth phases and culture media. The calculation was performed using SAS JMP 15 software.

\section{Declarations}

\section{Acknowledgements}

The study was supported by the Fundamental Research Grant Scheme (FRGS) by the Ministry of Higher Education Malaysia (Grant reference code: FRGS/1/2019/STG03/UM/02/11) and L'Oreal-UNESCO For Women in Science National Award 2019 (Grant number: PV009-2019).

\section{Author contributions}

FMSDS is the MSc student conducted bench work, collected data, analysed data and drafted the article; LKH is a chemist and co-PI of the project, experimental design, advised on methods for detection and identification of VOCs, analysed data, wrote and corrected the article; CLC is a microbiologist, the PI of the project, developed the idea, experimental design, analysed data, wrote and corrected the article.

\section{Competing Interests Statement}

The authors declare no competing interest.

\section{References}

1. Magana, M., Chatzipanagiotou, S., Burriel, A. R., \& loannidis, A.. Inquiring into the gaps of Campylobacter surveillance methods. Veterinary Sciences, <background-color:\#FFCC66;bverticalalign:super;>4</background-color:\#FFCC66;bvertical-align:super;>, 36; doi:10.3390/vetsci4030036 (2017).

2. Chlebicz, A. \& Slizewska, K.. Campylobacteriosis, Salmonellosis, Yersiniosis, and Listeriosis as zoonotic foodborne diseases: a review. International Journal Environmental Research Public Health, 
<background-color:\#FFCC66;bvertical-align:super;>15</background-color:\#FFCC66;bverticalalign:super;>, 863; doi.org/10.3390/ijerph15050863 (2018).

3. Ricke, S.C. et al. Developments in rapid detection methods for the detection of foodborne Campylobacter in the United States. Front. Microbiol., <background-color:\#FFCC66;bverticalalign:super;>9</background-color:\#FFCC66;bvertical-align:super;>, 3280; doi: 10.3389/fmicb.2018.03280 (2019).

4. Burnham, P. \& Hendrixson, D. Campylobacter jejuni: collective components promoting a successful enteric lifestyle. Nature Reviews Microbiology, <background-color:\#FFCC66;bverticalalign:super;>16</background-color:\#FFCC66;bvertical-align:super;>, 551-565; doi.org/10.1038/s41579-018-0037-9 (2018).

5. Gundogdu, O. \& Wren, B.W.. Microbe profile: Campylobacter jejuni - survival instincts. Microbiology, <bvertical-align:super;>166</bvertical-align:super;>, 230-232. doi10.1099/mic.0.000906 (2020).

6. Tang, Y. et al. Characterization and prevalence of Campylobacter spp. from broiler chicken rearing period to the slaughtering process in Eastern China. Frontiers in Veterinary Science, <backgroundcolor:\#FFCC66;bvertical-align:super;>7</background-color:\#FFCC66;bvertical-align:super;>, 227; doi.org/10.3389/fvets.2020.00227 (2020).

7. Kaakoush, N. O., Castaño-Rodríguez, N., Mitchell, H. M., \& Man, S. M. Global epidemiology of Campylobacter infection. Clinical microbiology reviews, <background-color:\#FFCC66;bverticalalign:super;>28</background-color:\#FFCC66;bvertical-align:super;><bvertical-align:super;> (</bvertical-align:super;><background-color:\#C8BE84;bvertical-align:super;>3</backgroundcolor:\#C8BE84;bvertical-align:super;><bvertical-align:super;>)</bvertical-align:super;>, 687-720; doi.org/10.1128/CMR.00006-15(2015).

8. Marder, E. et al.. Incidence and trends of infections with pathogens transmitted commonly through food and the effect of increasing use of culture-independent diagnostic tests on surveillance foodborne diseases active surveillance network, 10 U.S. sites, 2013-2016. U.S. Department of Health and Human Services Centers for Disease Control and Prevention, Morbidity and Mortality Weekly Report Weekly, <background-color:\#FFCC66;bvertical-align:super;>66</backgroundcolor:\#FFCC66;bvertical-align:super;>, 15; https://www.cdc.gov/mmwr/cme/conted_info.html\#weekly (2017).

9. Bessa, M.C. et al. Survey of Campylobacter in foods implicated in foodborne diseases in Southern Brazil. Revista do Instituto de Medicina Tropical de São Paulo, <bvertical-align:super; $>62</$ bverticalalign:super;>; doi.org/10.1590/S1678-9946202062090 (2020).

10. World Health Organization. The global view of campylobacteriosis: report of an expert consultation, Utrecht, Netherlands, 9-11 July 2012; http:/ apps.who.int/iris/bitstream/10665/.../1/9789241564601_eng.pdf (2013).

11. Sheppard, S.K. \& Maiden, M.C.J. The evolution of Campylobacter jejuni and Campylobacter coli. Cold Spring Harbor Perspectives in Biology, <bvertical-align:super;>7</bvertical-align:super;>, a018119; doi.10.1101/cshperspect. a018119 (2015). 
12. Igwaran, A. \& Okoh, A.I. Human campylobacteriosis: a public health concern of global importance. Heliyon, <background-color:\#FFCC66;bvertical-align:super;>5</background-color:\#FFCC66;bverticalalign:super;><bvertical-align:super;>(</bvertical-align:super;><background-color:\#C8BE84;bverticalalign:super;>11</background-color:\#C8BE84;bvertical-align:super;><bvertical-align:super;>) </bvertical-align:super;>, e02814; doi.org/10.1016/j.heliyon.2019.e02814 (2019).

13. Mortada, M., Cosby, D.E., Shanmugasundaram, R., Selvaraj, K. In vivo and in vitro assessment of commercial probiotic and organic acid feed additives in broilers challenged with Campylobacter coli. J. Appl. Poult. Res., <background-color:\#FFCC66;bvertical-align:super;>29</backgroundcolor:\#FFCC66;bvertical-align:super;>, 435-446; doi.org/10.1016/j.japr.2020.02.001 (2020).

14. Mikulic, M. et al. Prevalence of thermotolerant Campylobacter spp. in chicken meat in Croatia and multilocus sequence typing of a small subset of Campylobacter jejuni and Campylobacter coli isolates. Food Technology Biotechnology, <background-color:\#FFCC66;bverticalalign:super;>4</background-color:\#FFCC66;bvertical-align:super;>, 475-481; doi.org/10.17113/ftb.54.04.16.4647 (2016).

15. Wagenaar, J. A. et al. Campylobacter fetus infections in humans: exposure and disease. Clinical Infectious Diseases, <background-color:\#FFCC66;bvertical-align:super; $>11</$ backgroundcolor:\#FFCC66;bvertical-align:super;>, 1579-1586; doi.org/10.1093/cid/ciu085 (2014).

16. Zeng, D., Chen, Z., Jiang, Y., Xue, F. \& Li, B. Advances and challenges in viability detection of foodborne pathogens. Frontiers in Microbiology, <background-color:\#FFCC66;bverticalalign:super;>7</background-color:\#FFCC66;bvertical-align:super;>, 1833; dx.doi.org/10.3389\%2Ffmicb.2016.01833 (2016).

17. Buss, J. E. et al. Campylobacter culture fails to correctly detect Campylobacter in $30 \%$ of positive patient stool specimens compared to non-cultural methods. European journal of clinical microbiology \& infectious diseases, <background-color:\#FFCC66; bverticalalign:super;>38</background-color:\#FFCC66;bvertical-align:super;><bvertical-align:super;> (</bvertical-align:super;><background-color:\#C8BE84;bvertical-align:super;>6</backgroundcolor:\#C8BE84;bvertical-align:super;><bvertical-align:super;>)</bvertical-align:super;>, 1087-1093; doi.org/10.1007/s10096-019-03499-x (2019).

18. Ferone, M., Gowen, A., Fanning, S., Scanell, A.G.M. Microbial detection and identification methods: bench top assays to omics approaches. Comprehensive Reviews in Food Science and Food Safety, <background-color:\#FFCC66;bvertical-align:super;>19</background-color:\#FFCC66;bverticalalign:super;>, 3106-3129; doi.org/10.1111/1541-4337.12618 (2020).

19. Chen, J., Tang, J., Shi, H., Tang, C. \& Zhang, S. Characteristics of volatile organic compounds produced from five pathogenic bacteria by headspace-solid phase micro-extraction/gas chromatography-mass spectrometry. Journal of Basic Microbiology, <backgroundcolor:\#FFCC66;bvertical-align:super;>57</background-color:\#FFCC66;bvertical-align:super;>, 228237; doi.org/10.1002/jobm.201600505 (2017).

20. Karami N. et al. Resistant/susceptible classification of respiratory tract pathogenic bacteria based on volatile organic compounds profiling. Cell Mol. Biol., <background-color:\#FFCC66;bvertical- 
align:super;>64</background-color:\#FFCC66;bvertical-align:super;><bvertical-align:super;> $<$ /bvertical-align:super;><bvertical-align:super;>(</bvertical-align:super;><backgroundcolor:\#C8BE84;bvertical-align:super;>9</background-color:\#C8BE84;bvertical-align:super;><bverticalalign:super;>)</bvertical-align:super;>, 6-15; doi.org/10.14715/cmb/2018.64.9.2 (2018).

21. Smart, A. et al. Sniffing out resistance - rapid identification of urinary tract infection-causing bacteria and their antibiotic susceptibility using volatile metabolite profiles. Journal of Pharmaceutical and Biomedical Analysis, <background-color:\#FFCC66;bvertical-align:super;>167</backgroundcolor:\#FFCC66;bvertical-align:super;><bvertical-align:super;>,</bvertical-align:super;> 59-65; doi.org/10.1016/j.jpba.2019.01.044 (2019).

22. Kos, R. et al. Targeted exhaled breath analysis for detection of Pseudomonas aeruginosa in cystic fibrosis patients. Journal of Cystic Fibrosis; doi.org/10.1016/j.jcf.2021.04.015 (2021).

23. Hong-Geller, E. \& Adikari, S. Volatile organic compound and metabolite signatures as pathogen identifiers and biomarkers of infectious disease. Biosensing Technologies for the Detection of Pathogens; dx.doi.org/10.5772/intechopen.72398 (2017).

24. Garcia-Alcega, S. et al. Fingerprinting outdoor air environment using microbial volatile organic compounds (MVOCs) - a review. Trends in Analytical Chemistry, <backgroundcolor:\#FFCC66;bvertical-align:super;>86</background-color:\#FFCC66;bvertical-align:super;>, 75-83; doi.org/10.1016/j.trac.2016.10.010 (2017).

25. Wang, Y., Li, Y., Yang, J., Ruan, J. \& Sun, C. Microbial volatile organic compounds and their application in microorganism identification in foodstuff. Trends in Analytical Chemistry, <background-color:\#FFCC66;bvertical-align:super;>78</background-color:\#FFCC66;bverticalalign:super;>, 1-16; doi.org/10.1016/j.trac.2015.08.010 (2016).

26. Franchina, F. A., Purcaro, G., Burklund, A., Beccaria, M., Hill, J.E. Evaluation of different adsorbent materials for the untargeted and targeted bacterial VOC analysis using GCxGC-MS. Analytica Chimica Acta, <background-color:\#FFCC66;bvertical-align:super;>1066</backgroundcolor:\#FFCC66;bvertical-align:super;>, 146-153; doi.org/10.1016/j.aca.2019.03.027 (2019).

27. Matysik, S., Herbarth, O., Mueller, A. Determination of microbial volatile organic compounds (MVOCs) by passive sampling onto charcoal sorbents. Chemosphere, <background-color:\#FFCC66;bverticalalign:super;> 76</background-color:\#FFCC66;bvertical-align:super;>, 114-119; doi.org/10.1016/j.chemosphere.2009.02.010 (2009).

28. Ramirez, N., Cuadras, A., Rovira, E., Borrull, F., Marce, R.M. Comparative study of solvent extraction and thermal desorption methods for determining a wide range of volatile organic compounds in ambient air. Talanta, <background-color:\#FFCC66;bvertical-align:super;>82</backgroundcolor:\#FFCC66;bvertical-align:super;>, 719-727; doi.org/10.1016/j.talanta.2010.05.038 (2010).

29. Fang, S., Liu, S., Song, J., Huang, Q., Xiang, Z. Recognition of pathogens in food matrixes based on the untargeted in vivo microbial metabolite profiling via a novel SPME/GC $\times$ GC-QTOFMS approach. Food Research International, <background-color:\#FFCC66;bvertical-align:super;>142</backgroundcolor:\#FFCC66;bvertical-align:super;>, 110213; doi.org/10.1016/j.foodres.2021.110213 (2021). 
30. Weisskopf, L., Schulz, S. \& Garbeva, P. Microbial volatile organic compounds in intra-kingdom and inter-kingdom interactions. Nat Rev Microbiol., <background-color:\#FFCC66;bvertical-

align:super;>19</background-color:\#FFCC66;bvertical-align:super;>, 391-404; doi.org/10.1038/s41579-020-00508-1(2021).

31. Brondz, I. \& Olsen, I. Multivariate analyses of cellular fatty acids in bacteroides, Prevotella, Porphyromonas, Wolinella, and Campylobacter spp. Journal of clinical microbiology, <backgroundcolor:\#FFCC66;bvertical-align:super;>29</background-color:\#FFCC66;bvertical-align:super;> $<$ bvertical-align:super;>(</bvertical-align:super;><background-color:\#C8BE84;bverticalalign:super;>1</background-color:\#C8BE84;bvertical-align:super;><bvertical-align:super;>)</bverticalalign:super;>, 183-189; doi.org/10.1128/jcm.29.1.183-189 (1991).

32. Probert, C., Jones, P. \& Raticliffe, N. A novel method for rapidly diagnosing the causes of diarrhea. Small Intestine, <background-color:\#FFCC66;bvertical-align:super; $>53</$ backgroundcolor:\#FFCC66;bvertical-align:super;>, 58-61; doi.org/10.1136/gut.53.1.58 (2003).

33. Garner, C. et al. Identification of Campylobacter infection in chickens from volatile faecal emissions. Biomarkers, <background-color:\#FFCC66;bvertical-align:super;>13</backgroundcolor:\#FFCC66;bvertical-align:super;><bvertical-align:super;>(</bvertical-align:super;><backgroundcolor:\#C8BE84;bvertical-align:super;>4</background-color:\#C8BE84;bvertical-align:super;><bverticalalign:super;>)</bvertical-align:super;>, 413-421; doi.org/10.1080/13547500801966443 (2008).

34. Nunez-Carmona, E., Abbatangelo, M., \& Sberveglieri, V. Innovative sensor approach to follow Campylobacter jejuni development. Biosensors, <background-color:\#FFCC66;bverticalalign:super;>9</background-color:\#FFCC66;bvertical-align:super;><bvertical-align:super;>(</bverticalalign:super;><background-color:\#C8BE84;bvertical-align:super; $>1</$ backgroundcolor:\#C8BE84;bvertical-align:super;><bvertical-align:super;>)</bvertical-align:super;>, 8; dx.doi.org/10.3390/bios9010008 (2019).

35. Reese, K.L., Rasley, A., Avila, J.R., Jones, A.D., Frank, M. Metabolic profiling of volatile organic compounds (VOCs) emitted by the pathogens Francisella tularensis and Bacillus anthracis in liquid culture. Scientific Reports, <background-color:\#FFCC66;bvertical-align:super;>10</backgroundcolor:\#FFCC66;bvertical-align:super;>, 9333; doi.org/10.1038/s41598-020-66136-0 (2020).

36. Kelm, G.R. and Wickett, R.R. The role of fatty acids in cosmetic technology. Chapter 12 - the role of fatty acids in cosmetic technology, AOCS Press, 385-404; doi.org/10.1016/B978-0-12-8095218.00012-X (2017).

37. Audrain, B., Farag, M.A., Ryu, C., Ghigo, J. Role of bacterial volatile compounds in bacterial biology. FEMS Microbiology Reviews, <background-color:\#FFCC66;bvertical-align:super;>39</backgroundcolor:\#FFCC66;bvertical-align:super;><bvertical-align:super;></bvertical-align:super;><bverticalalign:super;><</bvertical-align:super;><background-color:\#C8BE84;bverticalalign:super;>2</background-color:\#C8BE84;bvertical-align:super;><bvertical-align:super;>)</bverticalalign:super;>, 222-233; doi.org/10.1093/femsre/fuu013 (2015).

38. Fillet, S., \& Adrio, J. L. Microbial production of fatty alcohols. World Journal of Microbiology \& Biotechnology, <background-color:\#FFCC66;bvertical-align:super;>32</background- 
color:\#FFCC66;bvertical-align:super;><bvertical-align:super;>(</bvertical-align:super;><backgroundcolor:\#C8BE84;bvertical-align:super;>9</background-color:\#C8BE84;bvertical-align:super;><bverticalalign:super;>)</bvertical-align:super;>, 152; doi.org/10.1007/s11274-016-2099-z (2016).

39. Sheoran, N. et al. Genetic analysis of plant endophytic Pseudomonas putida BP25 and chemoprofiling of its antimicrobial volatile organic compounds. Microbiological Research, <backgroundcolor:\#FFCC66;bvertical-align:super;>173</background-color:\#FFCC66;bvertical-align:super;>, 66-78; doi.org/10.1016/j.micres.2015.02.001(2015).

40. Lee, S., Shin, K., Lee, S. Exploring Nuruk Aroma; Identification of Volatile Compounds in Commercial Fermentation Starters. Food Science and Biotechnology, <background-color:\#FFCC66;bverticalalign:super;>25</background-color:\#FFCC66;bvertical-align:super;><bvertical-align:super;> (</bvertical-align:super;><background-color:\#C8BE84;bvertical-align:super;>2</backgroundcolor:\#C8BE84;bvertical-align:super;><bvertical-align:super;>)</bvertical-align:super;>, 393-399; https://dx.doi.org/10.1007\%2Fs10068-016-0054-2 (2016).

41. Fernando, W.G.D., Ramarathnam, R., Krishnamoorthy, A.S., Savchuk, S.C. Identification and use of potential bacterial organic antifungal volatiles in biocontrol. Soil Biology \& Biochemistry, <bverticalalign:super;>37</bvertical-align:super;>, 955-964; doi0.1016/j.soilbio.2004.10.021(2005).

42. Kim S. et al. PubChem 2019 update: improved access to chemical data. Nucleic Acids Res, <bvertical-align:super;>47</bvertical-align:super;><bvertical-align:super;>(</bvertical-align:super;> $<$ bvertical-align:super;>D</bvertical-align:super;><bvertical-align:super;>1</bvertical-align:super;> <bvertical-align:super;>)</bvertical-align:super;><bvertical-align:super;>,</bvertical-align:super;>11021109; doi:10.1093/nar/gky1033 (2019).

43. Karabagias, IK. A targeted metabolomic procedure for the identification of isophorone related compounds in honey. Journal of Plant Biochemistry \& Physiology, <bverticalalign:super;>6</bvertical-align:super;>, 1; doi10.4172/2329-9029.1000210 (2018).

44. Vidal, D.M., Moreira, M.A.B., Coracini, M.D.A., Zarbin, P.H.G. Isophorone derivatives as a new structural motif of aggregation pheromones in Curculionidae. Scientific Reports, <bverticalalign:super;>9</bvertical-align:super;>, 776; doi10.1038/s41598-018-37156-8 (2019).

45. Schulz, S. \& Dickschat, J.S. Bacterial volatiles: the smell of small organisms. Nat. Prod. Rep., <background-color:\#FFCC66;bvertical-align:super;>24</background-color:\#FFCC66;bverticalalign:super;>, 814-842; dx.doi.org/10.1039/b507392h (2007).

46. Awouafack, M.D., Tane, P., Kuete, V., Eloff J. N. Sesquiterpenes from the medicinal plants of Africa. Medicinal Plant Research in Africa, 1st edition, chapter 2, 33-103; dx.doi.org/10.1016/B978-0-12405927-6.00002-3 (2013).

47. Zhao, B. et al. Biosynthesis of the sesquiterpene antibiotic albaflavenone in Streptomyces coelicolor A3(2). Journal of Biological Chemistry, <bvertical-align:super;>283</bvertical-align:super;><bverticalalign:super;>(</bvertical-align:super;><bvertical-align:super;>13</bvertical-align:super;><bverticalalign:super;>)</bvertical-align:super;>, 8183-8189; doi10.1074/jbc.M710421200 (2008). 
48. Hidayati, S., Satyajaya, W., Fudholi, A. Lignin isolation from black liquor from oil palm empty fruit bunch using acid. Journal of Materials Research and Technology, <backgroundcolor:\#FFCC66;bvertical-align:super;>9</background-color:\#FFCC66;bvertical-align:super;><bverticalalign:super;>(</bvertical-align:super;><background-color:\#C8BE84;bverticalalign:super;>5</background-color:\#C8BE84;bvertical-align:super;><bvertical-align:super;>)</bverticalalign:super;>, 11382-11391; doi.org/10.1016/j.jmrt.2020.08.023 (2020).

49. Goh, E. B., Baidoo, E. E., Keasling, J. D., \& Beller, H. R. Engineering of bacterial methyl ketone synthesis for biofuels. Applied and environmental microbiology, $<$ backgroundcolor:\#FFCC66;bvertical-align:super;>78</background-color:\#FFCC66;bvertical-align:super;> <bvertical-align:super;>(</bvertical-align:super;><background-color:\#C8BE84;bverticalalign:super;>1</background-color:\#C8BE84;bvertical-align:super;><bvertical-align:super;>)</bverticalalign:super;>, 70-80; doi.org/10.1128/AEM.06785-11 (2012).

50. Pattanaik, B., \& Lindberg, P. Terpenoids and their biosynthesis in cyanobacteria. Life, <backgroundcolor:\#FFCC66;bvertical-align:super;>5</background-color:\#FFCC66;bvertical-align:super;><bverticalalign:super;>(</bvertical-align:super;><background-color:\#C8BE84;bverticalalign:super;>1</background-color:\#C8BE84;bvertical-align:super;><bvertical-align:super;>)</bverticalalign:super;>, 269-293; doi:10.3390/life5010269 (2015).

51. Milanowski, M. et al. Profiling of VOCs released from different salivary bacteria treated with nonlethal concentrations of silver nitrate. Analytical Biochemistry, <background-color:\#FFCC66;bverticalalign:super;>578</background-color:\#FFCC66;bvertical-align:super;>, 36-44; doi.org/10.1016/j.ab.2019.05.007 (2019).

52. Zhu, J. \& Hill, J.E. Detection of Escherichia coli via VOC profiling using secondary electrospray ionization-mass spectrometry (SESI-MS). Food Microbiology, <background-color:\#FFCC66;bverticalalign:super;>34</background-color:\#FFCC66;bvertical-align:super;>, 412-417; doi.org/10.1016/j.fm.2012.12.008 (2013).

53. Chai, L.C. et al. Thermophilic Campylobacter spp. in salad vegetables in Malaysia. International Journal of Food Microbiology, <background-color:\#FFCC66;bvertical-align:super;>117</backgroundcolor:\#FFCC66;bvertical-align:super;>, 106-111; doi.org/10.1016/j.ijfoodmicro.2007.02.014 (2007).

54. Jahiddin, F.S.A \& Low, K.H. Evaluation of terpene variability in the volatile oils from Zingiber officinale using chemometrics. Current Analytical Chemistry, <background-color:\#FFCC66;bverticalalign:super;>16</background-color:\#FFCC66;bvertical-align:super;><bvertical-align:super;> (</bvertical-align:super;><background-color:\#C8BE84;bvertical-align:super;>6</backgroundcolor:\#C8BE84;bvertical-align:super;><bvertical-align:super;>)</bvertical-align:super;>, 695-702; doi.org/10.2174/1573411015666190710221141 (2020).

55. U.S. EPA. Most probable number (MPN) calculator version 2.0. User and System Installation and Administration Manual. U.S. Environmental Protection Agency, Washington,DC;https://cfpub.epa.gov/si/si_public_record_Report.cfm? Lab=NERL\&dirEntryld=309398 (2013). 
56. Koo, I., Kim, S., Zhang, S. Comparative analysis of mass spectral matching-based compound identification in gas chromatography-mass spectrometry. Journal of Chromatography A, <background-color:\#FFCC66;bvertical-align:super;>1298</background-color:\#FFCC66;bverticalalign:super;>, 132-138; doi.org/10.1016/j.chroma.2013.05.021 (2013).

57. Van Den Dool, H. \& Kratz, P.D. A generalization of the retention index system including linear temperature programmed gas-liquid partition chromatography. Journal of Chromatography, <background-color:\#FFCC66;bvertical-align:super;>11</background-color:\#FFCC66;bverticalalign:super;>, 463-471; doi.org/10.1016/S0021-9673(01)80947-X (1963).

\section{Figures}
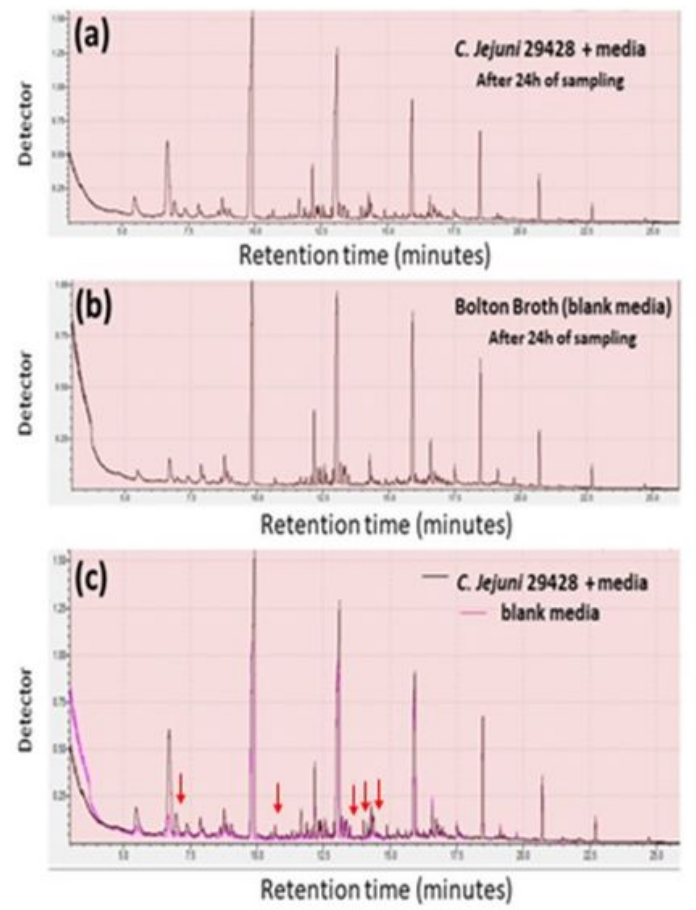

\section{Figure 1}

Comparison between chromatograms. Note: (a) C. jejuni 29428 after 24 hours of incubation and sampling, (b) negative control (blank Bolton Broth), and (c) overlap of chromatograms with the negative control peaks coloured in pink; the red arrows in part (c) represent the peaks that were not detected in the blank media. 


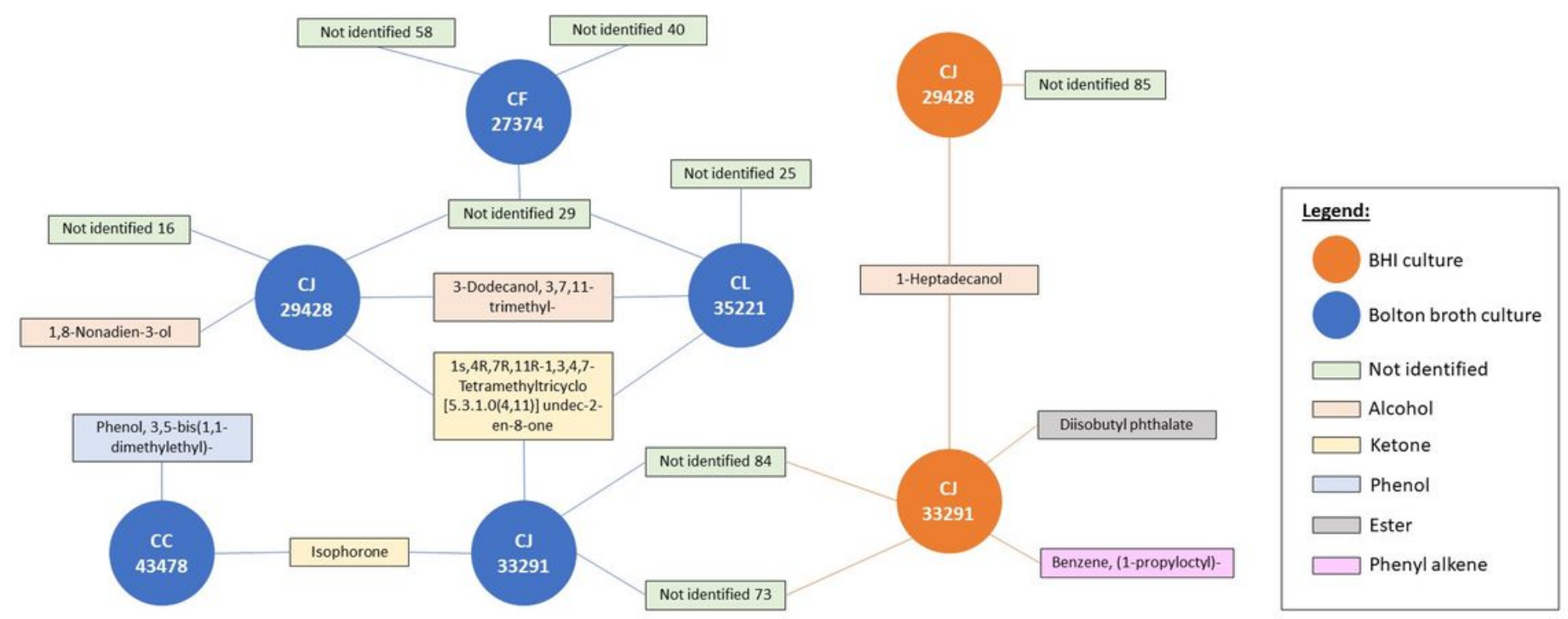

Figure 2

VOC biomarkers produced by C. jejuni (CJ) ATCC 29428, C. jejuni ATCC 33291, C. coli (CC) ATCC 43478, C. Iari (CL) ATCC 35221, C. fetus (CF) ATCC 27374 cultured in Bolton broth and BHI broth for 48 hours. 

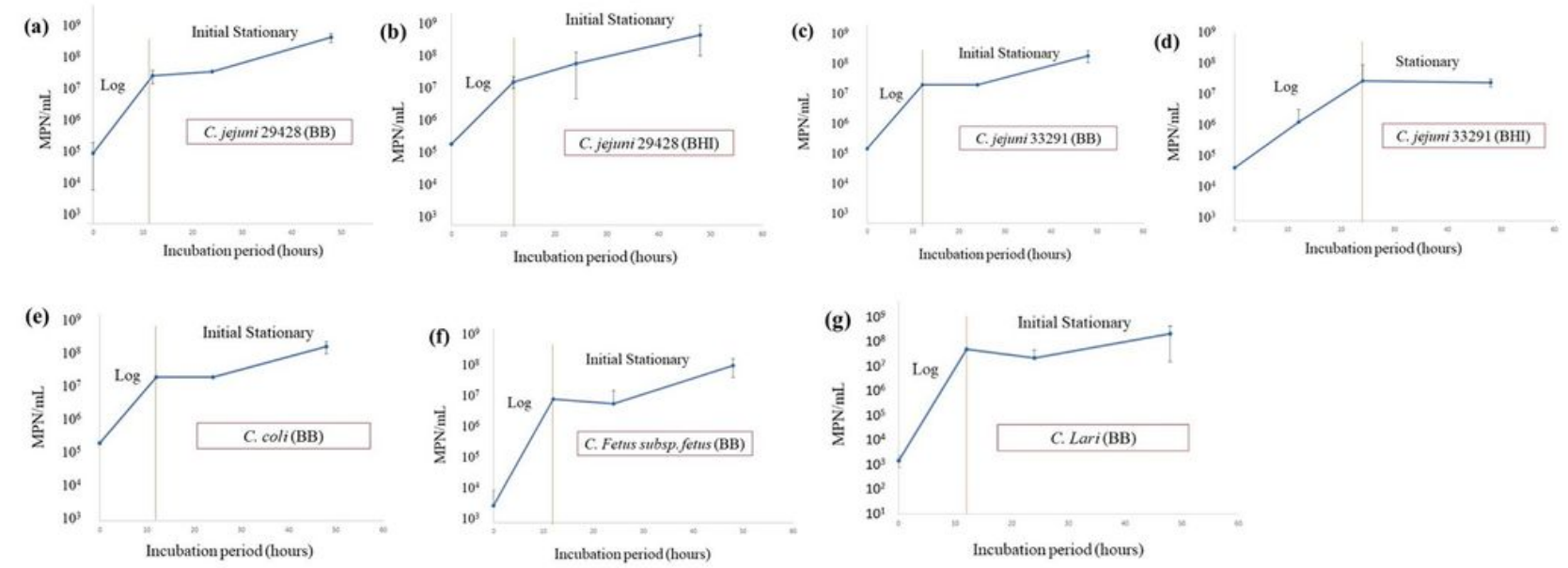

\section{Figure 3}

Growth curves in MPN (Most Probable Number) per mL of Campylobacterspp. (a) C. jejuni ATCC 29428 in Bolton broth (BB), (b) C. jejuni ATCC 29428 in BHI, (c) C. jejuni ATCC 33291 in BB, (d) C. jejuni ATCC 33291 in BHI, (e) C. coli ATCC 43478, (f) C. fetus subsp. fetus ATCC 27374, and (g) C. lari ATCC 35221, over incubation periods of $0,12,24$, and 48 hours throughout all repetitions. The error bars represent the standard deviation calculated within the MPN/mL of all the 5 repetitions at each timepoint. 

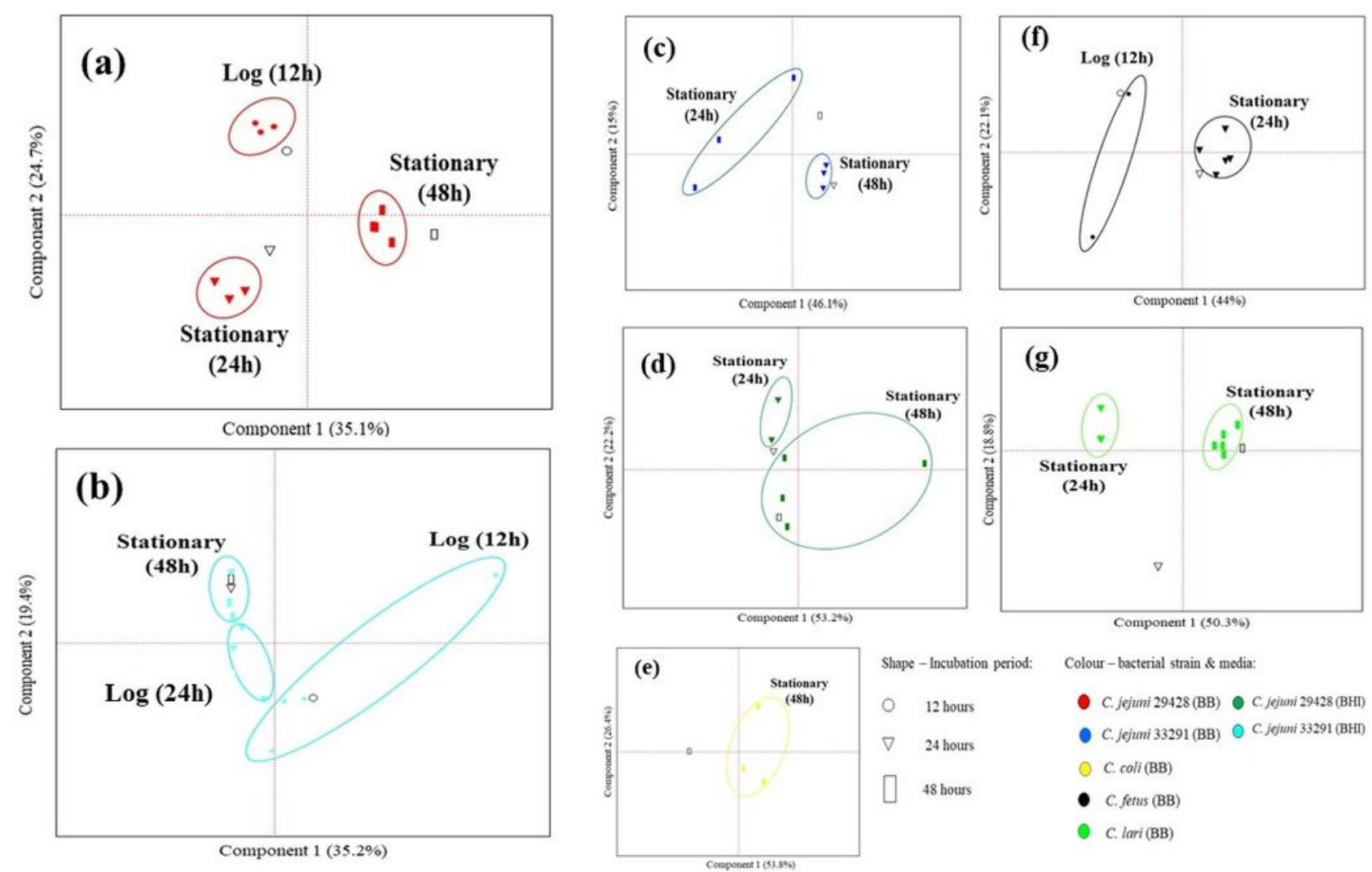

\section{Figure 4}

PCA score plots of each strain of Campylobacter at all incubation intervals with detected putative VOCs. Note: (a) C. jejuni 29428 (BB); (b) C. jejuni 33291 (BHI); (c) C. jejuni 33291 (BB); (d) C. jejuni 29428 (BHI); (e) C. coli; (f) C. fetus, (g) C. lari; each point represents a bacterial sample with detected VOC-markers; non-coloured shape represents the blank media (negative control). 


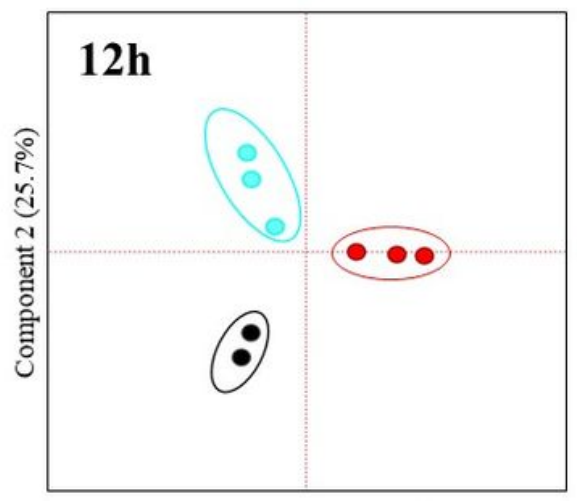

Component $1(41.4 \%)$

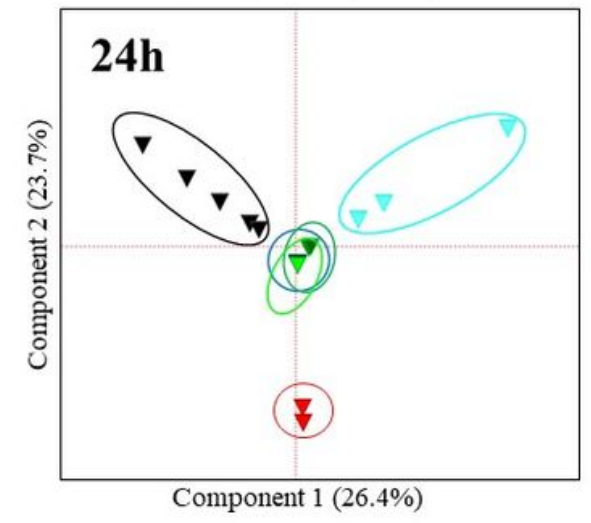

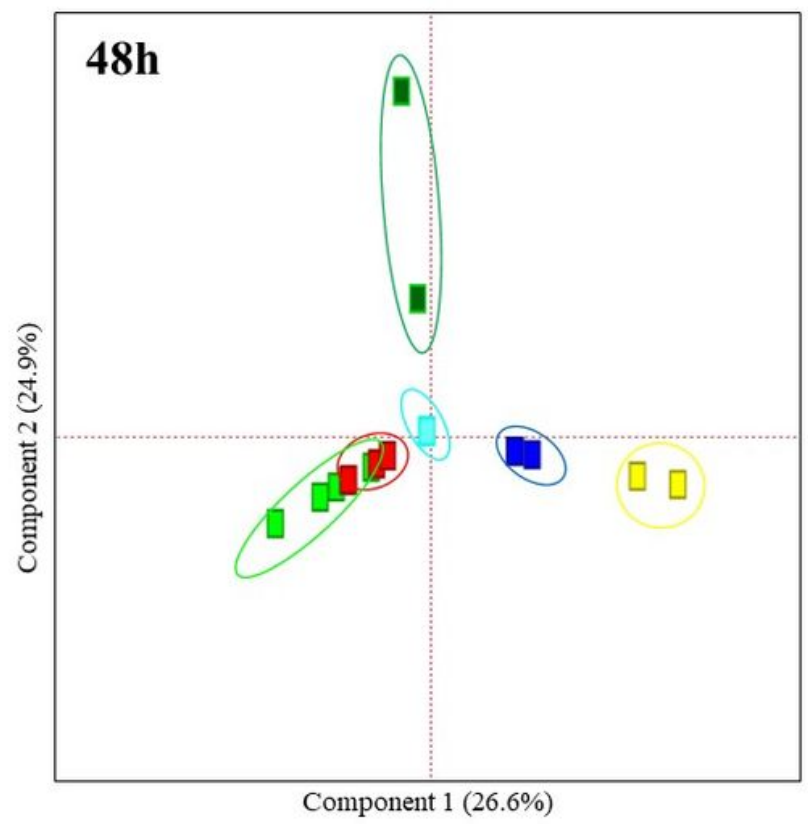

Shape - Incubation period: Colour - bacterial strain \& media:

12 hours

$\nabla \quad 24$ hours

48 hours
C. jejuni $29428(\mathrm{BB}) \bigcirc$ C. jejuni $29428(\mathrm{BHI})$
C. jejuni $33291(\mathrm{BB}) \bigcirc$ C. jejuni $33291(\mathrm{BHI})$
C. coli $\bigcirc$. fetus $\bigcirc$ C. lari

\section{Figure 5}

PCA score plots of all bacterial samples divided by each incubation period ( $12 \mathrm{~h}, 24 \mathrm{~h}$, and $48 \mathrm{~h})$. Note: each point represents a bacterial sample with its detected VOC-markers; the strains absent in one of intervals means that no putative VOC-markers were identified in these strains in that interval.

\section{Supplementary Files}

This is a list of supplementary files associated with this preprint. Click to download.

- SupplementaryA1.pdf 